\title{
Explorando el núcleo del significado de los conceptos. Incidencia de las variables psicolingüísticas en una tarea de reconocimiento
}

\author{
Exploring the Core of Meaning of Concepts. Influence of Psycholinguistic \\ Variables in a Word Recognition Task
}

Tomas Torres Barbero*

Gastón Deniau De Langhe**

Matías Yerro Avincetto***

Jorge Vivas $* * * *$

\section{Resumen}

Aún no existe precisión acerca de cuál es la relación entre las variables psicolingüísticas y el núcleo del significado conceptual. Este trabajo explora la relación de la Edad de Adquisición, la Imaginabilidad, la Riqueza semántica, la Familiaridad y la Concretud en la constitución del núcleo del significado. Se utiliza una tarea de reconocimiento con claves, construida con 120 conceptos y sus primeros 5 atributos extraídos de las Normas de Atributos Semánticos en Español. Participaron 300 estudiantes que debieron recuperar, en una combinatoria de 5 tomados de a 3, la etiqueta verbal del concepto al que tales atributos definen. Los mejores predictores fueron la EdA y la imaginabilidad. Estos resultados son consistentes con los estudios que sugieren la EdA como la variable que mejor explica el desempeño en tareas de Decisión Léxica.

Palabras clave: Núcleo del significado, Memoria semántica, Variables psicolingüísticas, Edad de Adquisición

\section{Abstract}

There still lacks preciseness about the nature of the connection between psycholinguistic variables and the core of conceptual meaning. This article explores the relation between Age of Acquisition (AoA), Imaginability, Semantic richness, Familiarity and Concreteness in the constitution of semantic core meaning. We used a key recognition task, designed with 120 concepts and their first 5 attributes, extracted from the

*Universidad Nacional de Mar del Plata. Facultad de Psicología ; Consejo Interuniversitario Nacional. Becas de Estimulo a las Formaciones Científicas. Mail de contacto:

torresbarberotomas@gmail.com

**Universidad Nacional de Mar del Plata. Facultad de Psicología

***Universidad Nacional de Mar del Plata. Facultad de Psicología

****Consejo Nacional de Investigaciones Científicas y Técnicas de Argentina. Universidad Nacional de Mar del Plata. Instituto de Psicología Básica, Aplicada y Tecnología

DOI: https://doi.org/10.46553/RPSI.17.33.2021.p61-80

Fecha de recepción: 14 de enero de 2021 - Fecha de Aceptación: 17 de marzo de 2021 
62 Explorando el núcleo del significado de los conceptos. Incidencia de las variables psi...

Semantic Attributes Norms in Spanish. 300 students took part in a concept recognition task, where they were asked to recognize certain concepts through different triads of attributes. The most influential variables were AoA and Imaginability. These results are congruent with studies that suggest the AoA as the most appropriate variable to explain results in lexical decisions tasks.

Key words: Core of meaning, Semantic memory, Psycholinguistic variables, Age of Acquisition

\section{Introducción}

Los seres humanos han adquirido durante la evolución una sofisticada capacidad para activar selectivamente representaciones mentales entre miles de etiquetas verbales y comprender sutiles matices de significado. De este modo, es posible decodificar un objeto con alta precisión a partir de muy poca información, ya que las pistas verbales usualmente desencadenan una representación coherente del mismo (Kivisaari et al., 2019). Entender cómo la gente se comprende a pesar de atribuir diversos significados a los conceptos expresados lexicalmente, requiere investigar las propiedades constitutivas de los atributos que permiten el acceso al significado conceptual.

Hay consenso en que un concepto se define a partir de los atributos utilizados para definirlo (McRae et al., 2005; Sartori, Polezzi, et al., 2005). Dichos atributos y los conceptos a los que se vinculan constituyen una parte sustantiva de la memoria semántica. En 1974, Smith et al. propusieron el Modelo de Comparación de Rasgos para modelar la organización de este tipo de memoria. Sugirieron que el significado de una palabra se constituye a través de la lista de atributos o rasgos definitorios.

Según este modelo, la operación para estimar el grado de "definibilidad" que tiene cada atributo es la siguiente: ante una oración se calcula un índice de semejanza de significado entre los conjuntos de atributos del sujeto y el predicado, y según el valor alcanzado los resultados posibles son tres: $\mathrm{Si}$ el valor obtenido excede un criterio umbral, se toma una decisión por la positiva - el atributo es definitorio de tal concepto- $y$ si el valor resulta por debajo del umbral, se decide por la negativa - atributo no asociado. Si el índice de semejanza adquiere un valor intermedio, ocurre una segunda etapa del proceso de atribución de significado donde se comparan los rasgos del sujeto con los del predicado: si el conjunto de los rasgos del predicado queda incluido en el conjunto de rasgos del sujeto, se decide por verdadero, en otro caso se decide por la negativa (Smith et al., 1974).

Estos atributos definitorios son piezas esenciales para establecer una comprensión entre hablantes y se ha observado una considerable estabilidad del núcleo del significado para conceptos concretos en distintas comunidades lingüísticas y grupos etarios (Vivas, Kogan, et al., 2020; Kremer y Baroni, 2011; García Coni et al., 2020; Vivas, Martínez, et al., 2020).

El Núcleo de significado conceptual

Como las palabras forman parte de los estímulos más ampliamente utilizados en la psicología cognitiva, la neuropsicología, la neurociencia cognitiva y la psicolingüística, se han diseñado varias técnicas para capturar sus atributos. La más comúnmente utilizada se denomina Feature Listing Task (FLT) 
y consiste en solicitar a los participantes de una misma comunidad lingüística que proporcionen una lista de atributos que, según su buen saber y entender, mejor definen a un concepto dado.

En los últimos 15 años los resultados de esta actividad se han compilado en Normas de Producción de Atributos Semánticos para diferentes lenguas (Buchanan et al., 2012; Buchanan et al., 2019; De Deyne et al., 2008; Deng et al., 2021; Devereux et al., 2014; Lenci et al., 2013; McRae et al., 2005; Moldovan et al., 2015; Montefinese et al., 2014; Ruts et al., 2004; Stein y de Azevedo Gomes, 2009; Vinson y Vigliocco, 2008; Vivas, Vivas, Comesaña, et al., 2017). Se considera que las Normas reflejan la estructura estadística de las categorías en estudio y dan cuenta de los aspectos de significado que son compartidos por una comunidad lingüística, dado que contienen distribuciones de frecuencia de atributos y otros datos estadísticos respecto a los conceptos.

Estas Normas revisten una irrenunciable relevancia para el estudio tanto de aspectos conceptuales como aplicados. En lo teórico, resuelven el problema referido por McRae et al. (2005) acerca de la falta de estimaciones cuantitativas derivadas empíricamente $\mathrm{y}$, en lo práctico, ofrecen un corpus de datos empíricos normalizados sobre los cuales trabajar en el diseño de tareas experimentales.

Dentro de las Normas observamos que la distribución de los atributos no es uniforme (Vivas et al., 2021), sino que existe: un gran grupo de atributos compartido por todos los individuos, los cuales están más fuertemente ligados al concepto $\mathrm{y}$ constituyen el nivel nuclear, otro grupo de atributos compartidos parcialmente, que no proveen información suficiente para distinguir diferencialmente el concepto $\mathrm{y}$, por último, los utilizados por muy pocos sujetos de manera personal e idiosincrática. Aquellos atributos mayormente compartidos conforman lo que se denomina núcleo del significado conceptual (Kremer y Baroni, 2011; Vivas, Martínez, et al., 2020; Vivas et al., 2019; Vivas, Montefinese, et al., 2020).

A estos rasgos definitorios se les denomina nucleares debido a que poseen la información mínima necesaria para identificar diferencialmente un concepto (Sartori y Lombardi, 2004) y son aquellos que la mayoría de los sujetos de una muestra nombró como definitorio de tal concepto en las Normas de atributos, por ejemplo, "perro" y "ladra". Para explorar el nivel nuclear del significado, se ha realizado un estudio preliminar y dos experimentos (Vivas et al., 2021) donde se puso a prueba la velocidad de reconocimiento, estimando que la presencia de atributos nucleares manifestaría una recuperación y reconocimiento más rápida y eficiente del concepto, que aquellos en los que se presenta sólo atributos parcialmente compartidos o idiosincráticos.

$\mathrm{Si}$ bien podría considerarse que el núcleo del significado conceptual posee variabilidad acorde a la comunidad lingüística y contexto sociocultural que se observe (Manoiloff et al., 2010), un reciente trabajo realizado con hablantes nativos de Inglés, Italiano y Español (Vivas, Montefinese, et al., 2020) sugiere que la caracterización de los conceptos concretos resulta estable en los tres idiomas, sustentando así el concepto de núcleo de significado semántico como el conglomerado de información fundamental, independiente de la variabilidad que diferencia a las comunidades lingüísticas. 
Por otro lado, la literatura sugiere que pueden observarse algunas particularidades en cuanto a la organización semántica de adultos mayores (AM) con respecto a la población de adultos jóvenes (AJ). Mediante un estudio donde se comparó la riqueza y distancia semántica entre AJ y AM, Vivas, Martínez, et al. (2020) encontraron una fuerte estabilidad en el núcleo conceptual, y diferencias en la fluidez. Por su parte, un estudio realizado por el Aging Lexicon Project encontró diferencias estructurales en una tarea de asociación libre, en la composición de la organización del lexicón y de su cohesión y densidad (Dubossarsky et al., 2017; Wulff et al., 2019). Complementariamente, se realizó la comparación del núcleo de significado en dos distintos tipos de tarea: una tarea de asociación de palabras y una tarea producción de atributos frente a un concepto (Vivas et al., 2019). Se observó una coincidencia del $72.5 \%$ en las respuestas en ambos tipos de tarea.

Estos antecedentes indican la presencia de un núcleo semántico relativamente estable, que conglomera los atributos con la información mínima para la identificación diferencial del concepto, siendo el proceso de recuperación aquel implicado en la reactivación de aquellos atributos con mayor grado de aporte a su significado.

Como concepto teórico, el Núcleo del significado conceptual presenta aún problemas no resueltos. No hay consenso claro acerca del límite que separa la información nuclear de los niveles compartido e idiosincrático, y tampoco se encuentra establecida la manera en que el mismo evoluciona a lo largo del desarrollo.
Para Collins y Loftus (1975) "no hay ningún atributo que sea absolutamente necesario para ninguna categoría”. Barsalou (2003) también se muestra pesimista con respecto a la presencia de un núcleo de significado conceptual, ya que define al contexto circundante del concepto recuperado como lo determinante para su significación y recuperación, bajo el argumento de que no se recuperan conceptos aisladamente, sino situados "en un contexto en que el objeto adquiere relevancia".

Sin embargo, Sartori et al. (2004) ofrecen una categorización a partir de la definición de núcleo anteriormente citada, que fundamenta la noción de núcleo semántico como uno de los tres niveles posibles de contribución al significado. La investigación empírica desarrollada en los últimos años sustenta el postulado de que algunos atributos son más importantes que otros cuando se trata de ofrecer una definición diferencial de un concepto (Montefinese et al., 2014), estableciendo así un continuo desde aquellos atributos más centrales para tal definición, hasta aquellos más idiosincráticos.

En esta línea, Borghesani y Piazza (2017) marcan la importancia de estudiar las variables subyacentes que clasifican los tipos de atributos y de conceptos para establecer cuál de ellas tiene mayor poder predictivo respecto al grado de contribución del atributo al significado del concepto. Una de las categorías inherentes al concepto que los investigadores estudiaron en los últimos años para abordar dicha cuestión son el conjunto de variables psicolingüísticas.

Variables psicolingüísticas de los conceptos

Las variables psicolingüísticas son características clasificatorias de los 
conceptos estipulados en las Normas de Atributos Semánticos, que son utilizadas por los autores para explicar tiempos de respuesta y decisiones lexicales de las muestras poblacionales, y en base a ellos establecer una clasificación de los mismos (Capitani et al., 2003; González-Nosti et al., 2014; Vivas, Vivas, Comesaña, et al., 2017).

- Familiaridad: Esta propiedad del concepto es una medida subjetiva que refiere al conocimiento que el participante tiene del concepto, derivada de las oportunidades en las que el participante entra en contacto con él o piensa en él en la vida diaria. Se ha señalado que los puntajes de familiaridad son importantes predictores del tiempo de denominación de imágenes (Cuetos et al., 1999; Snodgrass y Yuditsky, 1996), y se ha sugerido que la familiaridad afecta el reconocimiento de palabras y el acceso léxico en español (Bargetto Fernández y Riffo Ocares, 2019). Refiere al nivel de cotidianidad en que un concepto se evoca o utiliza y debe discriminarse de la frecuencia léxica, que indica, para un corpus electrónico dado, la cantidad de apariciones de un término por millón de palabras.

- Edad de Adquisición (EdA): Esta variable se estipula en base a la edad en que un concepto se aprendió, de manera tanto verbal como escrita. A través del modelo conexionista, se generó la hipótesis de la flexibilidad de la red (Ellis y Lambon Raplh, 2000), según la cual las palabras aprendidas más tempranamente son aquellas que manifiestan una ventaja en el procesamiento, debido a que la red posee mayor plasticidad en etapas tempranas del entrenamiento (Juhasz et al., 2018). Esta variable está fuertemente emparentada con la familiaridad, ya que son las palabras más familiares y utilizadas las que más tempranamente se aprenden. La diferencia entre ambas es que la EdA muestra, según ciertos estudios, un mayor grado de influencia en tareas de decisión léxica y nominación de objetos (Morrison y Ellis, 1995). Esta variable puede estudiarse tanto de forma longitudinal, realizando evaluaciones en niños en edad de desarrollo y estimando cuándo un concepto se aprende acorde a su evocación a partir de imágenes, y de manera transversal, consultando a sujetos adultos sobre cuándo consideran que adquirieron el conocimiento de tal concepto (Álvarez y Cuetos, 2007). Sartori et al. (2005) señalan que ambos métodos son complementarios.

- Frecuencia Léxica: La frecuencia léxica, o frecuencia de uso de las palabras, es una de las variables más determinantes en su reconocimiento visual. Es un indicador objetivo que se calcula estableciendo la aparición de un término por millón de palabras en corpus electrónicos de gran magnitud. Tradicionalmente, en el caso de la lengua española, la Real Academia Española (RAE) ha puesto a disposición de cualquier interesado un recurso en línea, 
gratuito y accesible. Se trata de un banco de datos compuesto por cuatro conjuntos de documentos: el Corpus Diacrónico del Español (CORDE), el Corpus de Referencia del Español Actual (CREA), el Corpus del Español del Siglo XXI (CORPES XXI) y el Corpus del Diccionario Histórico (CDH). A partir de estudios realizados con tareas de recuperación y reconocimiento de palabras, existe una clara convicción de que los términos con alta frecuencia léxica se evocan con mayor rapidez que aquellas que el hablante nativo no utiliza frecuentemente o a las que no se expone cotidianamente.

- Riqueza semántica: se operacionaliza como Número de atributos y refiere a la cantidad de información que brinda el significado de una palabra, pudiendo constituir esta una gran cantidad de información relacionada -alto grado de riqueza semánticao un recuento más discreto de información asociada a tal concepto — baja riqueza semántica. Pexman et al. (2008) y Yap et al. (2011) definen tres dimensiones para la medición de esta variable: dispersión de la red del significado de la palabra, conceptos "vecinos" asociados y número de atributos.

- Imaginabilidad: Se refiere a la facilidad con la que una palabra genera una imagen mental (Barca et al., 2002). Como sugieren Kivisaari et al. (2019) y O'Kraven y Kanwisher (2000), el patrón de recuperación de etiquetas semánticas de tales objetos probablemente utiliza las mismas vías neuronales en que ocurre su procesamiento perceptivo, lo cual clasifican a través del concepto de "imaginería".

- Concretud: estimada en base a la distinción de objetos tangibles o abstractos (Amsel y Cree, 2013; Gilhooly y Logie, 1980; MartínezCuitiño et al., 2018).

$\mathrm{La}$ influencia de las variables antedichas en tareas semánticas ha sido ampliamente estudiada. Carroll y White (1973) fueron los primeros en postular que la edad en que adquirimos las palabras influencia su velocidad de procesamiento, de forma independiente a cualquier otra variable involucrada en el proceso. Gilhooly y Gilhooly (1979) encontraron que las palabras aprendidas más tempranamente eran las más fácilmente reconocidas. Sin embargo, los resultados obtenidos por Juhasz et al. (2018) sugieren que tanto las palabras más tempranamente adquiridas como las de mayor frecuencia léxica poseen influencia en reconocimiento y decisiones lexicales. La frecuencia léxica es una variable sumamente influyente en todo tipo de tareas lingüísticas (González-Nosti y Cuetos, 2009).

En tareas de recuerdo libre y reconocimiento, Dewhurst et al. (1998) encontraron que la frecuencia léxica facilitaba la respuesta solo en ciertas listas, pero en ninguna de las dos tareas se observaban efectos de la EdA, argumentando así que ambas variables tienen efectos diferentes en el recuerdo y reconocimiento. Existen estudios que contradicen los resultados previamente mencionados y postulan a 
otras variables como las más influyentes en tareas de reconocimiento y decisión léxica. Gardiner et al. (1988) sugieren, por su parte, que la familiaridad incide en mayor medida que la frecuencia léxica en tareas de reconocimiento.

No hay un acuerdo claro acerca de la relación entre las variables psicolingüísticas y el significado nuclear de los conceptos. Son escasos los estudios sobre el desempeño de tales variables en tareas de reconocimiento tras la descripción con conceptos y atributos basados en normas. En tareas similares, donde se evalúan este tipo de variables, los resultados no son concluyentes (González-Nosti y Cuetos, 2009). Frente a este problema, es necesario explorar la relación entre dichas variables y el significado nuclear, para contribuir a comprender el desarrollo y constitución del núcleo del significado conceptual.

\section{Objetivo general}

Explorar la relación entre las variables psicolingüísticas bajo estudio y el núcleo del significado conceptual.

\section{Objetivos específicos}

1. Estudiar la relación entre la cantidad de aciertos, entendidos estos como la coincidencia entre las respuestas de los sujetos y las establecidas en las normas de atributos semánticos, y las variables psicolingüísticas en estudio.

2. Estudiar la relación de aciertos y falsas alarmas con relación a la clasificación taxonómica a la que pertenece el objeto presentado.

3. Estudiar la distribución de las falsas alarmas.

\section{Metodología}

\section{Diseño}

El presente trabajo es exploratoriodescriptivo y el diseño es cuasi experimental, con selección de la muestra intencional, no aleatoria. Participaron un total de 300 adultos jóvenes universitarios, 233 mujeres $(77,66 \%)$ y 67 varones $(22,33 \%)$, con una media de edad de 25.5 años $(\mathrm{DE}=8,2)$ y un nivel socioeconómico medio.

\section{Materiales}

Se tomaron 120 de los 400 conceptos correspondientes a las Normas de producción de Atributos Semánticos en español (Vivas, Vivas, Comesaña, et al., 2017). Los conceptos seleccionados corresponden a diversas categorías accesorios, animales, construcciones, frutas, herramientas, instrumentos musicales, medios de transporte, muebles, prendas de vestir, utensilios y verduras ${ }^{1}$.

De dichos conceptos, se seleccionaron los cinco primeros atributos de acuerdo a su frecuencia de producción. Los antecedentes indican que en torno a ese número se encuentra el límite del núcleo del significado de los conceptos concretos (Vivas et al., 2019; Vivas, Martínez, et al., 2020; Vivas, Montefinese, et al., 2020).

Para cada concepto se conformaron diez tríadas de atributos, a partir de la combinatoria de cinco tomados de a tres. Con cada una de las diez variantes así resultantes se conformó una lista, correspondiendo cada ítem de la lista a uno de los 120 conceptos. Una posible limitación de la disposición de los atributos seleccionada es la introducción de cierto sesgo en la respuesta, ya que algunas tríadas 
estarían constituidas por atributos de mayor frecuencia léxica y otras por aquellos de menor frecuencia.

\section{Procedimiento}

La tarea diseñada es de nombramiento tras descripción. Las tríadas de atributos fueron utilizadas como los reactivos frente a los cuales los participantes debían escribir como respuesta la etiqueta verbal a la cual consideraban que dichos atributos definían mejor, pudiendo coincidir, o no, con el concepto al que estos atributos refieren en las Normas.

\section{Figura 1}

Ej. de trial en los cuestionarios (los tres primeros atributos según frecuencia de producción para el concepto ANANÁ)

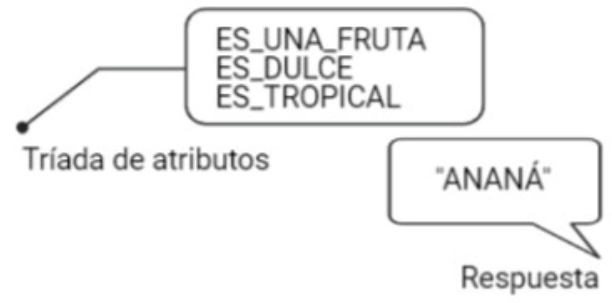

Es un tipo de tarea canónica, en donde se utiliza como estímulo una serie de atributos semánticos y el sujeto recupera y emite como respuesta la etiqueta referente a tales atributos, por medio del proceso cognitivo de reconocimiento. La evocación es un paso anterior al reconocimiento, constituye la recuperación de información a la consciencia desde la memoria a largo plazo por medio de asociación. Luego de evocados una serie de conceptos asociados a la tarea, se lleva a cabo la evaluación y exclusión de tales candidatos — se descartan aquellos que no provean información útil y se retienen aquellos que sí sean pertinentes para dar con el contenido buscado- $-y$, por último, se toma una decisión por uno de ellos dentro de toda la información disponible (Watkins y Gardiner, 1979).

La consigna mostrada en pantalla fue:

"A continuación, le presentaremos tríadas de atributos que refieren a un concepto que usted debe reconocer, por ej.: tiene manubrio, tiene dos ruedas, es un medio de transporte, en este caso, podría escribir "bicicleta" en el casillero correspondiente, y así sucesivamente con los demás conceptos que sean presentados. Algunos son тиy sencillos, otros menos, puede pensar en más de una respuesta, escriba siempre la que le parezca mejor. La tarea dura aproximadamente 40 minutos, es importante que lo haga en una sola sesión, pero no exige tiempo de respuesta, puede interrumpir ocasionalmente la actividad sin cerrar el programa y luego continuar. Pulse siguiente para iniciar la prueba."

Al comienzo de la tarea, se presentó un consentimiento informado donde constaban los objetivos del estudio y la tarea a realizar. Asimismo, se aclaró que los datos serían confidenciales y que los resultados solo serían utilizados con fines investigativos. No se solicitaron datos personales. Se aclaró lo dispuesto por la Ley Nacional Argentina de protección de los datos personales $\mathrm{N}^{\circ} 25.326$ y la "Guía de Conducta Ética en Ciencias Sociales y Humanas" desarrollada por el Comité de Ética del CONICET (Consejo Nacional de Investigaciones Científicas y Técnicas, Argentina), basada en los principios éticos y Código de Conducta para psicólogos de la 
APA de 2017.

La prueba duró aproximadamente 40 minutos y el formato de presentación fue digital. Se obtuvieron finalmente todas las respuestas de los sujetos a la presentación de las tríadas de atributos para los diez cuestionarios. Se enlistaron y unificaron en una hoja de cálculo todas las etiquetas conceptuales que los sujetos emitieron como respuestas. Luego, se consignó la frecuencia de aparición de tales etiquetas conceptuales y las mismas se clasificaron como "correctas" - aquellas que coinciden con el concepto al que tales atributos refieren en las normas-; como "falsas alarmas" — aquellos conceptos enunciados por los participantes que no coinciden con el concepto al que tales atributos refieren en las normas - y como fracasos - los casos en que no se pudo recuperar un concepto.

Para explorar la relación entre la correcta recuperación de la etiqueta verbal y sus variables psicolingüísticas asociadas, se realizó una regresión lineal considerando a la Familiaridad, Edad de Adquisición, Imaginabilidad, Concretud y Número de Atributos, como predictores de la cantidad de aciertos total por concepto. De esta forma, obtuvimos el coeficiente de determinación por parte de las variables bajo estudio sobre el total de respuestas correctas obtenidas. Los valores de las variables usadas fueron extraídos de las Normas de Producción de Atributos Semánticos en Español (Vivas, Vivas, Comesaña, et al., 2017).

Para explorar el desempeño relativo acorde a cada categoría semántica, se clasificaron los 120 conceptos según la categorización estipulada en las Normas y se asoció la misma con el porcentaje de aciertos, falsas alarmas y fallos para cada categoría.
Finalmente, para explorar la distribución de falsas alarmas, se seleccionaron los errores más frecuentes y se clasificaron los mismos de acuerdo a su similitud relativa con el concepto target. Paralelamente, se extrajo la frecuencia léxica disponible para tanto los falsos positivos como para los conceptos objetivo. Para ello se utilizó el LEXESP de Davis y Perea (2005), un software que permite obtener un amplio rango de datos estadísticos con respecto a conceptos en español, diseñado para investigaciones en psicolingüística. Dentro del amplio bagaje de datos que el software ofrece, se encuentra la frecuencia léxica de los conceptos, estimada en base al número de veces que un concepto aparece por millón de palabras.

\section{Resultados}

Respecto a la asociación entre variables psicolingüísticas y la correcta recuperación del concepto, se observa que la EdA obtuvo un ajuste sustancialmente distinto al resto de las variables, explicando en un $21 \%$ el acierto en las respuestas: para el total de aciertos y EdA, el análisis de regresión lineal arrojó un $\mathrm{R}^{2}$ de 0.21 , comparado con $\mathrm{R}^{2}=0.19$ en Imaginabilidad, $\mathrm{R}^{2}=0.03$ en Familiaridad y $\mathrm{R}^{2}=0.01$ en Concretud y Número de atributos.

En relación al éxito en la recuperación respecto a la categoría taxonómica a la que pertenecen cada uno de los conceptos, se observa un porcentaje de acierto de $82,16 \%$ para accesorios, $78,3 \%$ para medios de transporte, 70,91\% para construcciones, $67,01 \%$ para animales, $65,96 \%$ para frutas, $64,11 \%$ para muebles, $61,96 \%$ para utensilios, $60,45 \%$ para prendas de vestir, $60,11 \%$ para verduras, $58,83 \%$ para herramientas y 53,04\% para instrumentos 


\section{Figura 2}

Regresión lineal para total de aciertos por concepto en función de la Edad de Adquisición

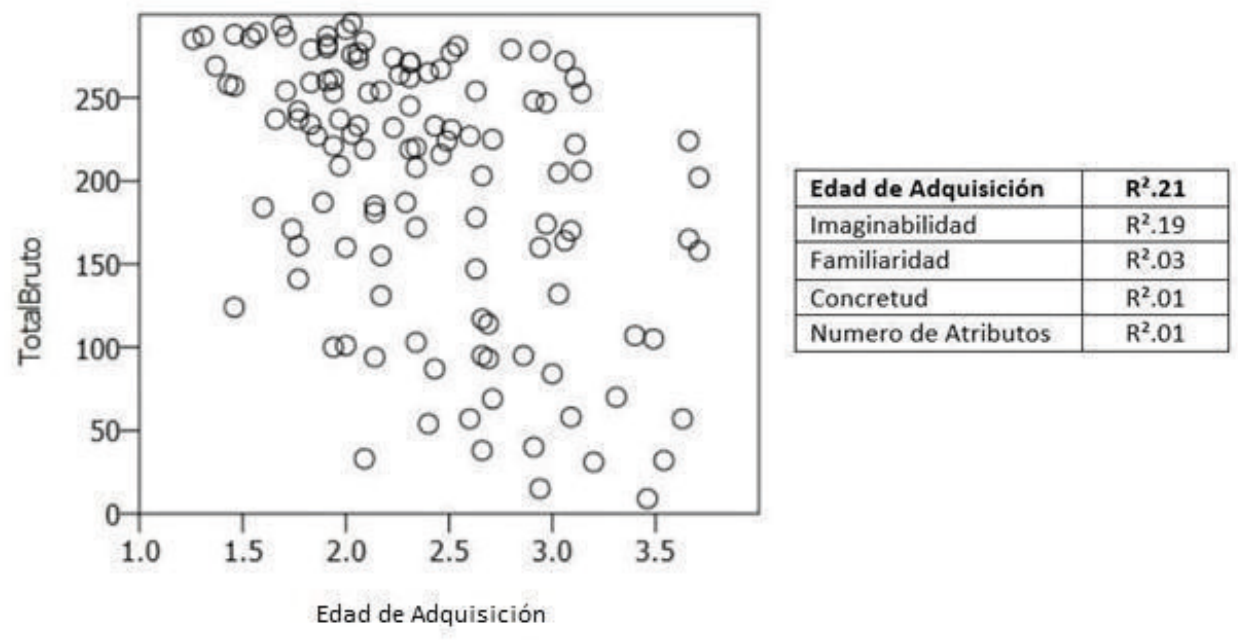

musicales.

En relación a la distribución de falsos positivos más frecuentes, la misma puede clasificarse en 4 conjuntos: (a) coordinados, es decir aquellos que conforman la misma categoría taxonómica que el concepto objetivo (por ejemplo, "maza" y" martillo", son conceptos coordinados bajo la categoría de herramientas); (b) sinónimos, aquellas etiquetas verbales equivalentes al término objetivo (Por ejemplo, para "banana", "plátano"). Para profundizar en el tema de la sinonimia, véase Zapico y Vivas (2015); (c) nombre de la categoría, aquellos casos en que el participante nombra la categoría general a la que pertenece el concepto y (d) respuestas idiosincráticas, que no guardan relación semántica directa con el objeto. Observamos una predominancia de conceptos inherentes a la misma categoría de pertenencia que el concepto objetivo $(76,42 \%)$; en menor cantidad respuestas idiosincráticas $(11,38 \%)$; casos de sinonimia $(9,75 \%) \mathrm{y}$, finalmente, categoría general $(2,43 \%)$.

Respecto a la diferencia de frecuencia léxica entre conceptos target y falsos positivos, se calculó el promedio de apariciones por millón para cada conjunto. El promedio de frecuencia léxica para conceptos target es de 12,83 mientras que el promedio en caso de los falsos positivos es de 21,48. Destacamos que en LEXESP no hay datos disponibles para un 7,05\% de los conceptos extraídos de las Normas; en caso de los falsos positivos más frecuentes, en esta base de datos no están presentes un $24,34 \%$ del total, por lo tanto, estos casos fueron excluidos del análisis.

De los resultados obtenidos, se puede inferir que cuando un sujeto debe responder frente a claves semánticas, existe preeminencia en la recuperación de conceptos más tempranamente aprendidos en su desarrollo y más fácilmente imaginables. 


\section{Figura 3}

Regresiones lineales para el total de aciertos por concepto en función de cada una de las variables psicolingüisticas en estudio
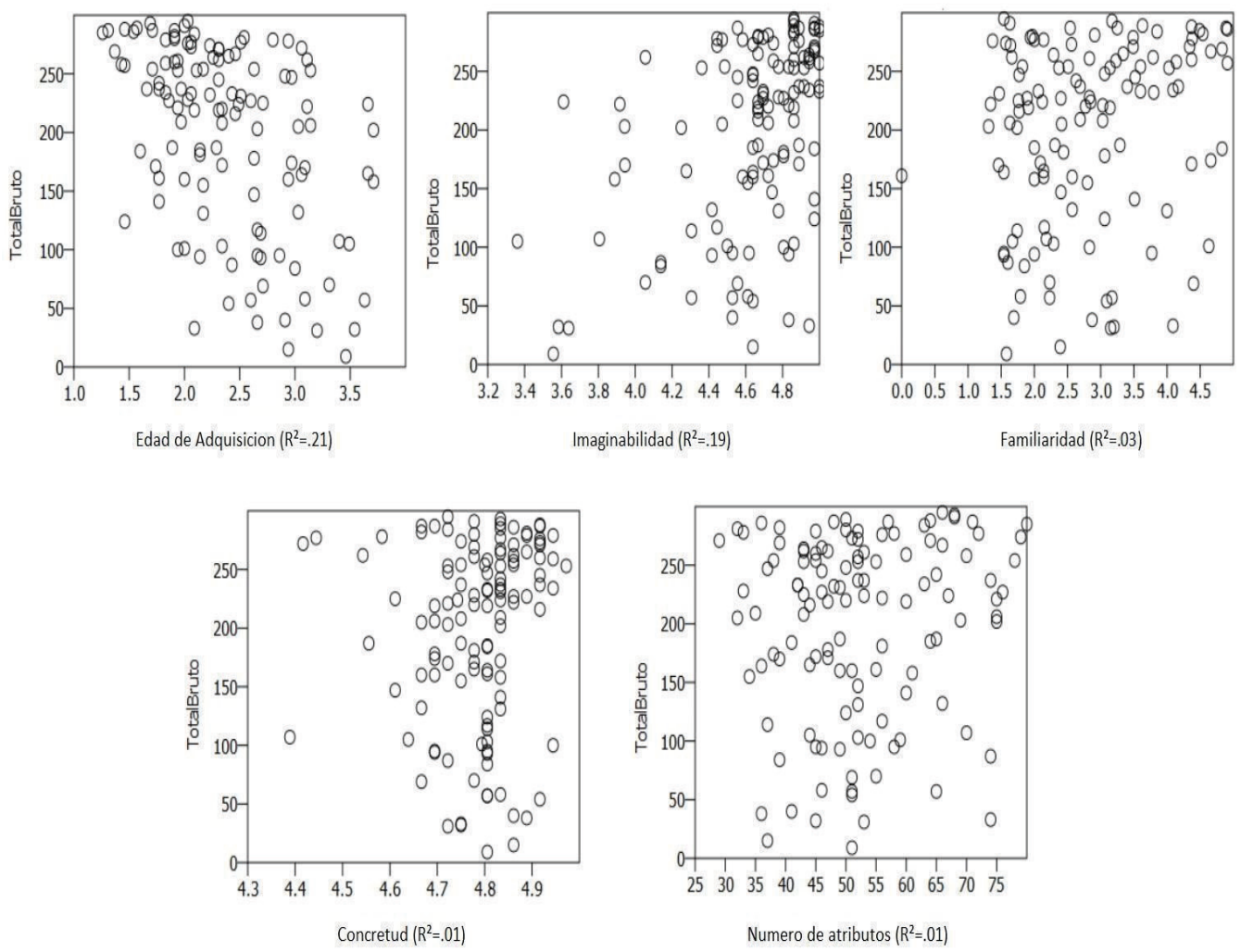

Sugerimos que la EdA se muestra como una de las variables psicolingüísticas con mayor influencia en la consolidación de los atributos que constituyen el significado nuclear de los conceptos, infiriendo que el núcleo del significado de los conceptos se constituye en las etapas más tempranas del desarrollo lingüístico. La observación de que las categorías de accesorios, animales y medios de transporte hayan mostrado una

mayor tasa de acierto respecto de las otras, sugiere que estos tipos de conceptos serán más fácilmente recuperados debido a la frecuencia del contacto y a las experiencias que el individuo haya establecido con los objetos de tal categoría, tanto a través del conocimiento enciclopédico como de la interacción cotidiana con el objeto, fundamentalmente en las primeras etapas del desarrollo. 
72 Explorando el núcleo del significado de los conceptos. Incidencia de las variables psi...

\section{Figura 4}

Porcentajes de acierto en la recuperación según categoría taxonómica de pertenencia

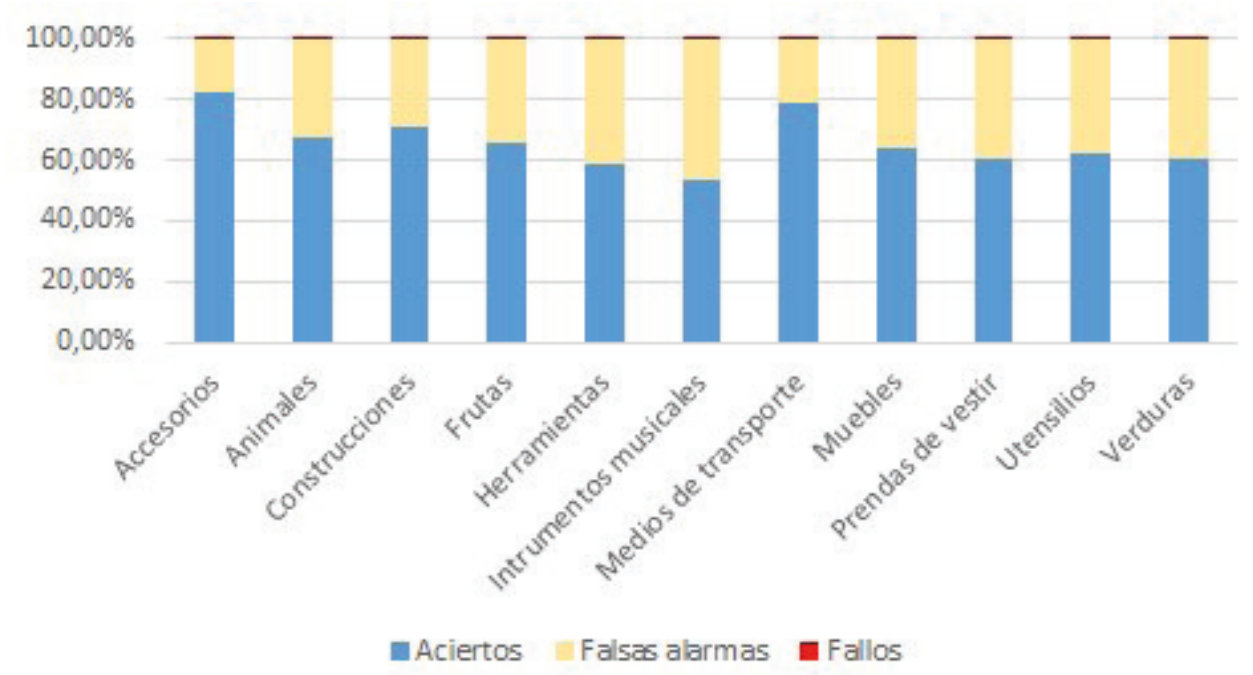

\section{Figura 5}

Distribución de falsos positivos según su relación con el concepto objetivo

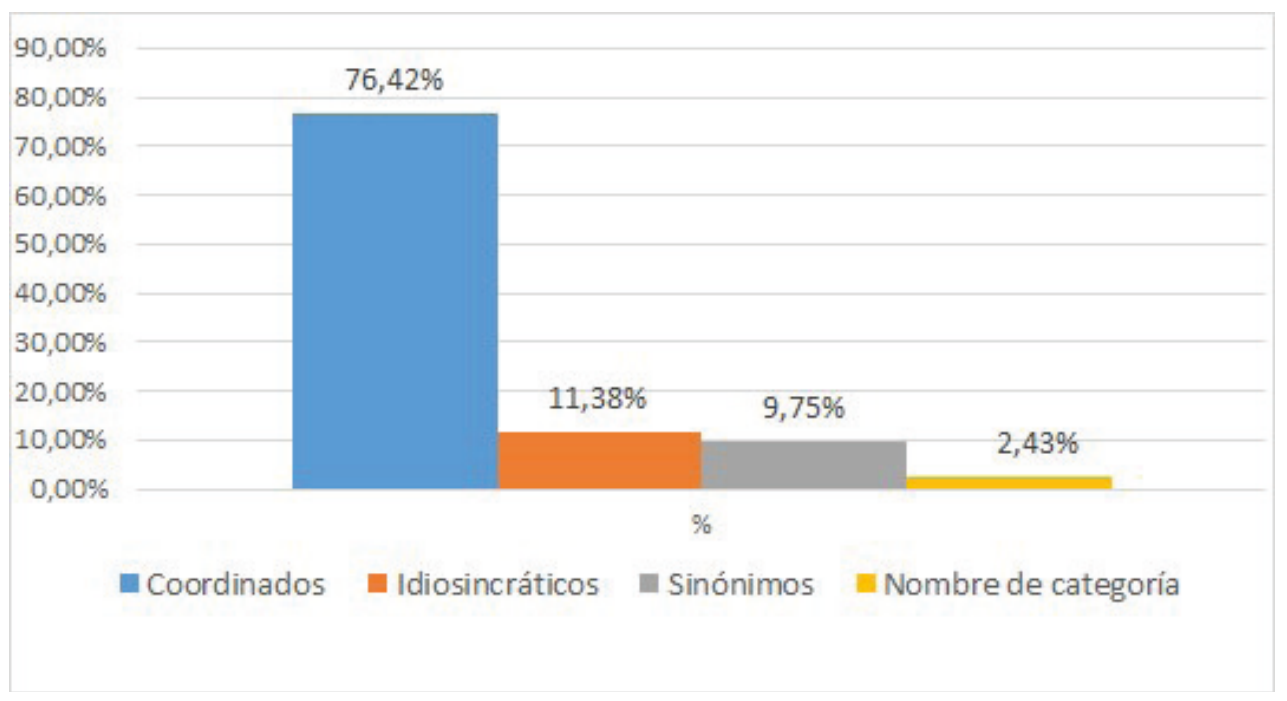

Revista de Psicología. Año 2021. Vol. 17, N 33, pp. 61-80 
Por último, observamos que en aquellos casos donde los atributos no proveen la información mínima suficiente para la recuperación del concepto, el significado nuclear resulta inaccesible. El individuo recupera principalmente conceptos adyacentes para los cuales resultan definitorios tales atributos comunes, es decir, se extrae el significado de conceptos coordinados bajo la misma categoría taxonómica, en base a la limitada información disponible.

\section{Discusión}

Entendiendo que el núcleo de significado de los conceptos está constituido por los atributos semánticos que permiten definir al concepto, comprenderse con los otros y a la vez diferenciarlo de los demás (Sartori y Lombardi, 2004; Vivas, Montefinese, et al., 2020), podemos sugerir que la edad en la que tales conceptos se aprendieron es uno de sus indicadores diferenciales. La relación observada entre el núcleo del significado conceptual y EdA como variable que influye en el proceso de recuperación, nos permite situar la categoría nuclear como constituida en las primeras etapas del desarrollo psicolingüístico del sujeto, sugiriendo que la información adquirida más tempranamente será fundamental para la construcción de la etiqueta conceptual.

En la medida en que la información ofrecida no sea suficiente para permitir el acceso al nivel nuclear del significado, la activación se dirigirá mayormente a palabras ligadas a la misma categoría taxonómica

\section{Figura 6}

Comparación entre promedios de frecuencia léxica para conceptos target y falsos positivos

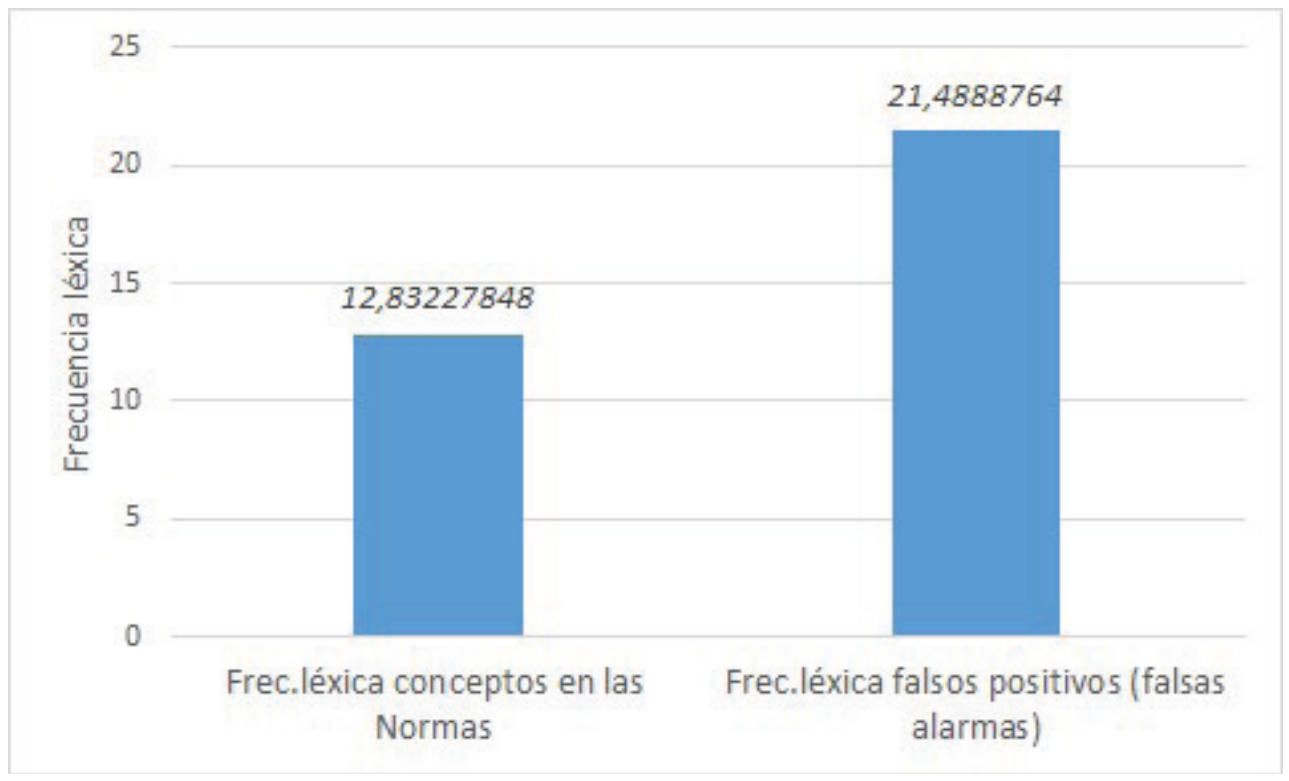


74 Explorando el núcleo del significado de los conceptos. Incidencia de las variables psi...

de pertenencia, y hacia aquellos conceptos de mayor frecuencia en el léxico de la comunidad lingüística a la que el sujeto pertenece. Sin embargo, podríamos pensar que esta última observación se relaciona a los efectos de variables psicolingüísticas como EdA, más que a la frecuencia léxica de manera aislada.

Se sugirió por mucho tiempo que la frecuencia léxica y la EdA son variables muy difíciles de aislar mutuamente, encontrando que en tareas de decisión léxica y reconocimiento de palabras, la mayor influencia parecía estar dada en la frecuencia léxica de los ítems utilizados. Aun así, en los últimos años la Edad de adquisición ha manifestado poder explicativo con respecto a otras variables sobre las que se ha estudiado más exhaustivamente: por ejemplo, se demostró que, en afasias adquiridas, las palabras anteriormente aprendidas en el desarrollo son más resistentes al deterioro que aquellas aprendidas de forma tardía (Brysbaert y Ellis, 2016). En otro estudio clínico realizado en pacientes multilingües, se observó que las palabras más tempranamente adquiridas son aquellas que mayor representación poseen en zonas corticales del procesamiento del lenguaje, principalmente la corteza perisilviana izquierda (Fernández-Coello et al., 2016).

Así pues, tal como sugiere la hipótesis semántica inicialmente planteada por Van Loon Vervoon y Van Der Koppen (1988), según la cual todo el conocimiento conceptual es dependiente del orden en que los conceptos fueron aprendidos a lo largo del desarrollo, hoy se sabe con certeza que muchos de los efectos atribuidos en el pasado a la frecuencia léxica, en realidad corresponden a la EdA (González-Nosti y
Cuetos, 2009; Morrison y Ellis, 1995). Este postulado se ve sustentado por resultados de estudios más recientes en tareas de nombramiento de palabras (Elsherif et al., 2019), lectura de frases (Juhasz et al., 2018), tiempos de respuesta (Brysbaert et al., 2000) y en tareas de reconocimiento (GonzálezNosti y Cuetos 2009); así como con los resultados obtenidos en el presente estudio. Por otro lado, observamos que la variable psicolingüística Imaginabilidad presenta un ajuste inferior a EdA, pero sustancialmente mejor al resto, lo cual podría explicarse porque el patrón de recuperación de etiquetas semánticas de objetos probablemente tiene lugar en las mismas vías neuronales en que ocurre el procesamiento perceptivo de tales objetos, como sugieren O'Kraven y Kanwisher (2000).

Kivisaari et al. (2019) utilizan una tarea parecida a la diseñada en el presente trabajo, y afirman que el proceso de recuperación activado, al solicitar la recuperación de una etiqueta conceptual a través de pistas semánticas, realmente no parece diferenciarse del proceso de Imaginabilidad, en donde se realiza la evocación de contenido latente en la imaginería a partir de alguno de los rasgos definitorios del objeto.

Este fenómeno se encuentra ampliamente explicado por Lawrence Barsalou (2003; 2009), por medio del concepto de simulación: cuando evocamos un objeto a nuestra memoria en ausencia de este, el sistema cognitivo elabora una simulación que constituye un símbolo perceptual (Barsalou, 1999), para el cual se activan los mismos patrones neuronales que se activaron al momento en que el objeto se encontraba presente. Esta simulación no 
es idéntica a tal objeto sino un prototipo de todos los ejemplares (Barsalou, 2008), construido a partir de información multimodal almacenada en la memoria a través de las experiencias sensoriales del sujeto. Finalmente, puede pensarse que las características centrales que las personas evocan para definir un concepto tal como se refleja en el corpus de información recabado en las Normas, tienen que ver con la simulación genérica compartida por una misma comunidad lingüística, construida por los atributos nucleares que otorgan el acceso al significado. Dado que los atributos utilizados poseen un orden de presentación distinto acorde al cuestionario, un punto de abordaje futuro sobre los datos del presente trabajo es estudiar el efecto de primacía (Hernández Muñoz, 2014; McRae y Boisvert, 1998) manifestado por el primer atributo de la triada y cómo influye el prime concatenado.

\section{Referencias}

Álvarez, B. y Cuetos, F. (2007). Objective age of acquisition norms for a set of 328 words in Spanish. BRM 39, 377383. https://doi.org/10.3758/ BF03193006

Amsel, B. D., \& Cree, G. S. (2013). Semantic richness, concreteness, and object domain: An electrophysiological study. Canadian Journal of Experimental Psychology/Revue canadienne de psychologie expérimentale, $\quad 67(2), \quad 117-$ 129. https://doi.org/10.1037/ a0029807

Barca, L. Burani, C. y Arduino, L.S (2002) Word naming times and Psycholinguistic norms for Italian nouns. BRMI\&C 34, 424434. https://doi.org/10.3758/ BF03195471

Barsalou, L.W. (2009) Simulation, situated conceptualization, and prediction. Phil. Trans. R. Soc. B, 364, 12811289. https://doi.org/10.1098/ rstb.2008.0319

Barsalou, L. (1999). Perceptual symbol systems. Behavioral and Brain Sciences, 22(4), 577-660.
doi:10.1017/S0140525X99002149

Barsalou, L. (2008) Grounded Cognition. Аnпu. Rev. Psy. (59), 617-645. https://doi.org/10.1146/annurev. psych.59.103006.093639

Barsalou, L. (2003) Situated simulation in the human conceptual system. Language and Cognitive Processes, 18:5-6, 513-562.https:// doi.org/10.1080/0169096 034000026

Bargetto Fernández, M. A. y Riffo Ocares, B. (2019). El reconocimiento de palabras y el acceso léxico: revisión de modelos y pruebas experimentales. Boletín de filología, 54(1), 341-361. https:// dx.doi.org/10.4067/S071893032019000100341

Borghesani, V. y Piazza, M. (2017). The neuro cognitive representations of symbols: The case of concrete words. Neuropsychologia, 105, 4-7. https://doi.org/10.1016/j. neuropsychologia.2017.06.026

Brysbaert, M. y Ellis, A. (2016) Aphasia and age of acquisition: are earlylearned words more resilient? 
76 Explorando el núcleo del significado de los conceptos. Incidencia de las variables psi...

Aphasia, Frequency and Language Processing 11 (30). https://doi.org/ 10.1080/02687038.2015.1106439

Brysbaert, M., Van Wijnendaele, I. y De Dayne, S. (2000) Age of acquisition effects in semantic processing tasks. Acta Psychologica, 104 (4), 215216.https://doi.org/10.1016/S00016918(00)00021-4

Buchanan, E., Valentine, K. y Maxwell, N. (2019). English semantic feature production norms: An extended database of 4436 concepts. BRM 51, 1849-1863. https://doi. org/10.3758/s13428-019-01243-Z

Buchanan, E., Holmes, J., Teasley, M. y Hutchison, K. (2012). English semantic wordpair norms and a searchable Web portal for experimental stimulus creation. $B R M, 45(3), 746-757$. https://doi. org/10.3758/s13428-012-0284-z

Capitani, E., Laiacona, M., Mahon, B. y Caramazza, A. (2003). What are the facts of semantic categoryspecific deficits? A critical review of the clinical evidence. Cognitive Neuropsychology, 20, 213-261. https://doi. org/10.1080/02643290244000266

Carroll, J.y White, M.(1973). Word frequency and age of acquisition as determiners of picture naming latency. Quart JEP 25, 85-95. https://doi. org/10.1080/14640747308400325

Collins, A. y Loftus, E. (1975). A spreadingactivation theory of semantic processing. Psychological Review, 82(6), 407-428. https://doi. org/10.1037/0033-295X.82.6.407

Cuetos, F., Ellis, A. y Álvarez, B. (1999).
Naming times for the Snodgrass and Vanderwart pictures in Spanish. BRM\&C 31, 650-658. https://doi. org/10.3758/BF03200741

Davis, C. J. y Perea, M. (2005)BuscaPalabras: A program for deriving orthographic and phonological neighborhood statistics and other psycholinguistic indices in Spanish. Behavior Research Methods. 37, 665-671. https://doi.org/10.3758/ BF03192738

Deng, Y., Wang, Y., Qiu, Ch., Hu, Z., Sun, W., Gong, Y., Zhao, X. He, W., y Cao, L. (2021) A Chinese Conceptual Semantic Feature Dataset (CCFD). $B R M$ (preprint). https://doi. org/10.3758/s13428-020-01525-X

De Deyne, S., Verheyen, S., Ameel, E., Vanpaemel, W., Dry, M. J., Voorspoels, W. y Storms, G. (2008). Exemplar by feature applicability matrices and other Dutch normative data for concepts. BRM 40, 10301048. https://doi.org/10.3758/ BRM.40.4.1030

Devereux, B., Tyler, L., Geertzen, J. y Randall, B. (2014). The Centre for Speech, Language and the Brain (CSLB) concept property norms. BRM 46, 1119-1127. https://doi. org/10.3758/s13428-013-0420-4

Dewhurst, S. A., Hitch, G. J. y Barry, C. (1998). Separate effects of word frequency and age of acquisition in recognition and recall. JEP: LM\&C 24(2), 284-298. https://doi. org $/ 10.1037 / 0278-7393.24 .2 .284$

Dubossarsky, H., De Deyne, S. y Hills, T. (2017). Quantifying the structure of free association networks across 
Explorando el núcleo del significado de los conceptos. Incidencia de las variables psi...

the life span. Developmental Psychology, 53(8), 1560 1570. https://doi.org/10.1037/ dev0000347

Ellis, A. y Lambon Ralph, M. (2000). Age of Acquisition Effects in Adult Lexical Processing Reflect Loss of Plasticity in Maturing Systems: Insights from Connectionist Networks. JEP: $L M \& C$ 26(2), 1103-1123. https://doi.org/10.1037/02787393.26.5.1103

Elsherif, M., Catling, J. y Frisson, S. (2020). Two words as one: A multinaming investigation of the ageof-acquisition effect in compoundword processing. Memory and Cognition 48, 511-525. https://doi. org/10.3758/s13421-019-00986-6

Fernández-Coello, A., Havas, V., Juncadella, M., Sierpowska, J., Rodríguez-Fornells, A. y Gabarrós A. (2017). Age of language acquisition and cortical language organization in multilingual patients undergoing awake brain mapping. Journal of Neurosurgery 126(6):1912-1923. $\quad$ https://doi. org/10.3171/2016.5.JNS152791

García Coni, A., Comesaña, A., Piccolo, B. y Vivas, J. (2020). Relaciones conceptuales: Comparación entre niños, adultos jóvenes y adultos mayores. RIP, 13 (1), 59-69. https:// reviberopsicologia.ibero.edu.co/ article/view/1617

Gardiner, J., Gregg, V. y Hampton, J. (1988). Word frequency and generation effects. JEP: $L, M$, and C, 14(4), 687-693. https://doi. org $/ 10.1037 / 0278-7393.14 .4 .687$
Gilhooly, K. y Gilhooly, M. (1979). Ageof-acquisition effects in lexical and episodic memory tasks. Memory and Cognition, 7(3), 214-223. https:// doi.org/10.3758/BF03197541

Gilhooly, K. \& Logie, R. (1980) Age-ofacquisition, imagery, concreteness, familiarity, and ambiguity measures for 1944 words. Behavior Research methods 12(4). 395-427 https://doi. org/10.3758/BF03201693

González-Nosti, M., Barbón, A., RodríguezFerreiro, J. y Cuetos, F. (2014). Effects of the psycholinguistic variables on the lexical decision task in Spanish: A study with 2,765 words. BRM 46, 517-525. https:// doi.org/10.3758/s13428-013-0383$\underline{5}$

González-Nosti， M. y Cuetos, F. (2009) Influencia de las variables psicolingüísticas sobre el efecto de generación. Psicológica, 30(2):323342. https://www.uv.es/psicologica/ articulos2.09/10GONZALEZ.pdf

Hernández Muñoz, N. (2014). Categorías en el léxico bilingüe: perspectivas desde el priming semántico y la disponibilidad léxica. RAEL (13) 1 , 19-38. Gale Academic OneFile.

Juhasz B, Yap M, Raoul A, Kaye M. (2018). A further examination of word frequency and age-of-acquisition effects in English lexical decision task performance: The role of muñofrequency trajectory. JEP: $L M \& C$ 45(1):82-96.

https://doi.org/10.1037/xlm0000564

Kivisaari, S., van Vliet, M., Hultén, A., LindhKnuutila, T., Faisal, A. y Salmelin, R. (2019). Reconstructing meaning 
78 Explorando el núcleo del significado de los conceptos. Incidencia de las variables psi...

from bits of information. Nature Communications 10, 927. https:// doi.org/10.1038/s41467-019$\underline{08848-0}$

Kremer, G., Baroni, M. (2011). A set of semantic norms for German and Italian. BRM 43, 97-109. doi: https://doi.org/10.3758/s13428010-0028-x

Lenci, A., Baroni, M., Cazzolli, G. y Marotta G. (2013). BLIND: a set of semantic feature norms from the congenitally blind. BRM 45(4):1218-33. https:// doi.org/10.3758/s13428-013-03234

Manoiloff, L., Artstein, M., Canavoso, M., Fernández, L. y Segui, J. (2010) Expanded norms for 400 experimental pictures in an Argentinean Spanish-speaking population. BRM 42(2):452460. https://doi.org/10.3758/ BRM.42.2.452

Martínez-Cuitiño, M., Soriano, F., Formoso, J., Borovinsky, G., Ferrari, J., Pontello, N., Barreyro, J.P. y Manes, F. (2018). Procesamiento semántico de conceptos concretos y abstractos en Afasia Progresiva Primariavariante semántica. Revista de Investigación en Logopedia 8(1) 2018: 63-76.

http://dx.doi.org/10.5209/RLOG.59530

McRae, K. y Boisvert, S. (1998). Automatic Semantic Similarity Priming. JEP: $L, M$, and $C$ (24) 3, 558572. https://doi.org/10.1037/02787393.24.3.558

McRae, K., Cree, G., Seidenberg, M. y McNorgan, C. (2005). Semantic feature production norms for a large set of living and nonliving things. BRM, 37(4), 547-559. https://doi. org $/ 10.3758 / \mathrm{BF} 03192726$

Moldovan, C., Ferré, P., Demestre, J. y Sánchez-Casas, R. (2015). Semantic similarity: normative ratings for 185 Spanish noun triplets. BRM 47(3), 788-799. https://doi.org/10.3758/ s13428-014-0501-Z

Montefinese, M., Ambrosini, E., Fairfield, B. y Mammarella, N. (2014). The adaptation of the Affective Norms for English Words (ANEW) for Italian. BRM, 46(3), 887-903. https://doi.org/10.3758/s13428013-0405-3

Morrison, C. M., y Ellis, A. W. (1995). Roles of word frequency and age of acquisition in word naming and lexical decision. JEP: $L, M$, and C, 21(1), 116-133. https://doi. org $/ 10.1037 / 0278-7393.21 .1 .116$

O'Craven K. y Kanwisher N. (2000). Mental imagery of faces and places activates corresponding stimulus-specific brain regions. Journal of Cognitive Neuroscience 12(6):1013-23. https://doi. org/10.1162/08989290051137549

Pexman, P., Hargreaves, I., Siakaluk, P. (2008). There are many ways to be rich: Effects of three measures of semantic richness on visual word recognition. Psychonomic Bulletin \& Review 15, 161-167. https://doi. org/10.3758/PBR.15.1.161

Ruts, W., De Deyne, S., Ameel, E., Vanpaemel, W., Verbeemen, T. y Storms, G. (2004). Dutch norm data for 13 semantic categories and 338 exemplars. $B R M \& C \quad 36(3)$, 
Explorando el núcleo del significado de los conceptos. Incidencia de las variables psi...

506-515. https://doi.org/10.3758/ BF03195597

Sartori, G., Lombardi, L. y Mattiuzzi, L. (2005). Semantic relevance best predicts normal and abnormal name retrieval. Neuropsychologia, 43(5), $754-770$. https://doi.org/10.1016/j. neuropsychologia.2004.08.001

Sartori, G., \& Lombardi, L. (2004). Semantic relevance and semantic disorders. Journal of Cognitive Neuroscience, 16, 439-452. doi:10.1016/j. neuropsychologia.2006.08.028

Sartori, G., Polezzi, D., Mameli, F. y Lombardi, L. (2005). Feature type effects in semantic memory: an event related potentials study. Neuroscience Letters, 390. 139144. 10.1016/j.neulet.2005.08.015

Smith, E., Shoben, E., y Rips, L. (1974). Structure and process in semantic memory: A featural model for semantic decisions. Psyc Rev 81(3), 214-241. https://doi.org/10.1037/ $\underline{\mathrm{h} 0036351}$

Snodgrass, J. y Yuditsky, T. (1996). Naming times for the Snodgrass and Vanderwart pictures. BRMI\&C 28(4), 516-536.

https://doi.org/10.3758/BF03200540

Stein, L. y de Azevedo Gomes, C. F. (2009). Normas brasileiras para listas de palavras associadas: associação semântica, concretude, frequência e emocionalidade. Teor. e Pesq. (25) 537-546. Doi: 10.1590/S010237722009000400009

Van Loon-Vervoorn A. y Van Der Koppen M.(1988). The Importance of Age of Word Acquisition for Imageability in Word Processing. In: Denis
M., Engelkamp J., Richardson J.T.E. (eds) Cognitive and Neuropsychological Approaches to Mental Imagery. https://doi. org/10.1007/978-94-009-1391-2_9.

Vinson, D.P. Vigliocco, G. (2008) Semantic feature production norms for a large set of concepts of objects and events. Behavior Research Methods (40) 183-190.

Vivas, J., Kogan, B., Romanelli, S., Lizarralde, F. y Corda, L. (2020). A cross-linguistic comparison of Spanish and English semantic norms: Looking at core features. Applied Psycholinguistics, 41(2), 285-297. https://doi. org/10.1017/S0142716419000523

Vivas, J., Kogan, B., Yerro, M., Romanelli, S. y Vivas, L. (2021). Levels in the structure of concepts. Describing the structure of concepts through different feature levels. Journal of Cognitive Psychology. Taylor \& Francis ISSN: 2044592X. https:// doi.org/10.1080/20445911.2020.18 58840

Vivas, J., Martínez, S., Krzemien, D. y Lizarralde, F. (2020). Estabilidad semántica en la producción de atributos semánticos entre adultos mayores y adultos jóvenes. APL $\quad 38(2), \quad 1-19 . \quad$ https://doi. org/10.12804/revistas.urosario.edu. co/apl/a.7368

Vivas, J. Vivas, L. y MacIntyre, M. (2017) Tipología de atributos ponderada en base a Normas de Atributos Semánticos. Trends in Psychology (25) 2, 843-854. http://dx.doi. org/10.9788/TP2017.2-21Es 
80 Explorando el núcleo del significado de los conceptos. Incidencia de las variables psi...

Vivas, J., Vivas, L., Comesaña, A., García Coni, A. y Vorano, A. (2017). Spanish semantic feature production norms for 400 concrete concepts. BRM, 49, 3, 1095-1106. doi: $10.3758 / \mathrm{s} 13428-016-0777-2$

Vivas, L. Manoiloff, L. Garcia, A. Lizarralde, F. y Vivas, J. (2019). Core semantic links or lexical associations: Assessing the nature of responses Word Association Tasks. Journal of psycholinguistic research 48(2), 243-256. https://doi.org/10.1007/ s10936-018-9601-8

Vivas, L., Montefinese, M., Bolognesi, M. y Vivas, J. (2020). Core features: measures and characterization for different languages. Cognitive Processing - International Quarterly of Cognitive Science. Special Topic. https://link.springer. com/article/10.1007\%2Fs10339020-00969-5This article is part of the Special Topic on 'Eliciting Semantic Properties: Methods and Applications' guest-edited by E. Canessa, S. Chaigneau, B. Devereux, and A. Lenci. Guest editor: Alessandro Lenci
(University of Pisa).

Watkins, M. y Gardiner, J. (1979). An appreciation of generate-recognize theory of recall. Journal of Verbal Learning \& Verbal Behavior, 18(6), 687-704. https://doi.org/10.1016/ $\underline{\mathrm{S} 0022-5371(79) 90397-9}$

Wulff, D., de Deyne, S., Jones, M. y Mata, R. (2019). New perspectives on the aging lexicón. Trends in cognitive sciences, 23(8), 686-698. https:// doi.org/10.1016/j.tics.2019.05.003

Yap, M., Tan, S., Pexman, P. y Hargreaves I. (2011). Is more always better? Effects of semantic richness on lexical decision, speeded pronunciation, and semantic classification. Psych Bull Rev 18, 742-750. https://doi.org/10.3758/ s13423-011-0092-y

Zapico, G. y Vivas, J. (2015). La sinonimia desde una perspectiva lingüísticocognitiva. Medición de la distancia semántica, Onomázein. Revista de Lingüistica, Filología y Traducción 32,198-211.http://www.onomazein. net/04 NumeroDescarga/n32/ PantallaDescarga 32-11.html

\section{Notas al final}

${ }^{1} \mathrm{Si}$ bien los conceptos seleccionados pertenecen todos a la categoría de concretos, incorporar al análisis la variable psicolingüística de Concretud resulta especialmente útil para observar en qué grado esta propiedad del concepto resulta específicamente facilitadora del procesamiento semántico $\mathrm{y}$, aunque se encuentran emparentados, la concretud no es idéntica al objeto concreto considerado como ente, ya que como nos indican los datos recabados en bases de datos como LEXESP (Davis y Perea, 2005), estos objetos varían en su grado de concretud. Los atributos semánticos del concepto a identificar pueden ser de cualquier tipo, tanto funcionales como perceptivos, asociativos o enciclopédicos. 